\title{
THE FROBENIUS POSTAGE STAMP PROBLEM, AND BEYOND
}

\author{
ANDREW GRANVILLE AND GEORGE SHAKAN \\ Dedicated to Endre Szemerédi on the occasion of his 80th birthday
}

\begin{abstract}
Let $A$ be a finite subset of $\mathbb{Z}^{n}$, which generates $\mathbb{Z}^{n}$ additively. We provide a precise description of the $N$-fold sumsets $N A$ for $N$ sufficiently large, with some explicit bounds on "sufficiently large."
\end{abstract}

\section{INTRODUCTION}

Let $A$ be a given finite subset of the integers. For any integer $N \geq 1$, we are interested in determining the $N$-fold sumset of $A$,

$$
N A:=\left\{a_{1}+\cdots+a_{N}: a_{1}, \ldots, a_{N} \in A\right\},
$$

where the $a_{i}$ 's are not necessarily distinct. For simplicity we may assume without loss of generality that the smallest element of $A$ is 0 , and that the gcd of its elements is 1$]$ Under these assumptions we know that

$$
0 \in A \subset 2 A \subset 3 A \subset \cdots \subset \mathbb{N},
$$

where $\mathbb{N}$ is the natural numbers, defined to be the integers $\geq 0$. Moreover there exist integers $m_{1}, \ldots, m_{k}$ such that $m_{1} a_{1}+\cdots+m_{k} a_{k}=1$, and therefore

$$
\mathcal{P}(A)=\left\{\sum_{a \in A} n_{a} a: \quad \text { Each } n_{a} \in \mathbb{N}\right\}=\lim _{N \rightarrow \infty} N A=\mathbb{N} \backslash \mathcal{E}(A)
$$

for some finite exceptional set $\mathcal{E}(A)$ ?

One very special case is the Frobenius postage stamp problem in which we wish to determine what exact postage cost one can make up from an unlimited of $a$ cent and $b$ cent stamps. In other words, we wish to determine $\mathcal{P}(A)$ for $A=\{0, a, b\}$. It is a fun challenge for a primary school student to show that $\# \mathcal{E}(\{0,3,5\})=\{1,2,4,7\}$, and more generally, Sy1884], that

$$
\max \mathcal{E}(\{0, a, b\})=a b-a-b \text {, and }|\mathcal{E}(\{0, a, b\})|=\frac{1}{2}(a-1)(b-1) .
$$

Erdős and Graham [ErGr72] conjectured precise bounds for max $\mathcal{E}(A)$; see also Dixmier Di90.

A.G. was funded by the European Research Council grant agreement $\mathrm{n}^{\circ} 670239$, and by the Natural Sciences and Engineering Research Council of Canada (NSERC) under the Canada Research Chairs program. G.S. was supported by Ben Green's Simons Investigator Grant 376201. Many thanks to Seva Lev and Tyrrell McAllister for pointing us to the references [SaChe07] and [SiTi03, respectively.

${ }^{1}$ Since if we translate $A$ then we translate $N A$ predictably, as $N(A+\tau)=N A+N \tau$, and since if $A=g \cdot B:=\{g b: b \in B\}$ then $N A=g \cdot N B$.

${ }^{2}$ We give a simple proof that $\mathcal{E}(A)$ is finite in section 1.1. 
In this article we study the variant in which we only allow the use of at most $N$ stamps; that is, can we determine the structure of the set $N A$ ? If $b=\max A$, then $N A \subset\{0, \ldots, b N\} \cap \mathcal{P}(A)=\{0, \ldots, b N\} \backslash \mathcal{E}(A)$. Moreover, we can use symmetry to determine a complementary exceptional set: Define the set $b-A:=\{b-a: a \in A\}$. Then $N A=N b-N(b-A)$ and so $N A$ cannot contain any elements $N b-e$ where $e \in \mathcal{E}(b-A)$. Therefore

$$
N A \subset\{0, \ldots, b N\} \backslash(\mathcal{E}(A) \cup(b N-\mathcal{E}(b-A))) .
$$

We ask when equality holds?

Theorem 1 Let $A$ be a given finite subset of the integers, with smallest element 0 and largest element $b$, in which the gcd of the elements of $A$ is 1 . If $N \geq 2\left[\frac{b}{2}\right]$ and $0 \leq n \leq N b$ with $n \notin \mathcal{E}(A) \cup(N b-\mathcal{E}(b-A))$ then $n \in N A$. Equivalently, we have

$$
N A=\{0, \ldots, b N\} \backslash(\mathcal{E}(A) \cup(b N-\mathcal{E}(b-A))) .
$$

In the next section we will show that if $A$ has just three elements then Theorem 1 holds for all integers $N \geq 1$ (which does not seem to have been observed before). However this is not true for larger $A$ : If $A=\{0,1, b-1, b\}$ then $\mathcal{E}(A)=\mathcal{E}(b-A)=\emptyset$ and $b-2 \in(b-2) A$ but $b-2 \notin(b-3) A$, in which case Theorem 11 can only hold for $N \geq b-2$. We conjecture that one should be able to obtain the lower bound " $N \geq b-2$ " (which would then be best possible) in place of " $N \geq 2\left[\frac{b}{2}\right]$ " in Theorem 1 13 It is feasible that one could develop our methods to show this, but it seems to us like that would be a formidable task.

Theorem 1 seems to have first been proved, but with the bound $N \geq b^{2}(\# A-1)$, by Nathanson [Nat72] in 1972, which was improved to $N \geq \sum_{a \in A, a \neq 0}(a-1)$ in [WCC11] 4

We will generalize Theorem 1 to sets $A$ of arbitrary dimensions. Here we assume that $0 \in A \subset \mathbb{Z}^{n}$. The convex hull of the points in $A$ is given by

$$
H(A)=\left\{\sum_{a \in A} c_{a} a: \sum_{a \in A} c_{a}=1, \text { each } c_{a} \geq 0\right\},
$$

so that

$$
C_{A}:=\left\{\sum_{a \in A} c_{a} a: \text { Each } c_{a} \geq 0\right\}=\lim _{N \rightarrow \infty} N H(A),
$$

is the cone generated by $A$. Let $\mathcal{P}(A)$ be the set of sums in $C_{A}$ where each $c_{a} \in \mathbb{N}$, so that $\mathcal{P}(A) \subset C_{A} \cap \mathbb{Z}^{n}$. We define the exceptional set to be

$$
\mathcal{E}(A):=\left(C_{A} \cap \mathbb{Z}^{n}\right) \backslash \mathcal{P}(A),
$$

the integer points that are in the convex hull of positive linear combinations of points from $A$, and yet are not an element of $N A$, for any integer $N \geq 1$. With this notation we can formulate our result:

\footnotetext{
${ }^{3}$ Bearing in mind the example $A=\{0,1, N+1, N+2, \ldots, b\}$, we can refine this conjecture to " $N \geq b+2-\# A$ " whenever \#A $\geq 4$.

"WCC11] claim that their result is "best possible," but this is a consequence of how they formulate their result. Indeed Theorem 1 yields at least as good a bound for all sets $A$ with $\# A \geq 4$, and is better in all but a couple of families of examples.
} 
Theorem 2 Let $0 \in A \subset \mathbb{Z}^{n}$ such that $A$ spans $\mathbb{Z}^{n}$ as a vector space over $\mathbb{Z}$. There exists a constant $N_{A}$ such that if $N \geq N_{A}$,

$$
N A=\left(N H(A) \cap \mathbb{Z}^{n}\right) \backslash \mathcal{E}_{N}(A) \text { where } \mathcal{E}_{N}(A):=\left(\mathcal{E}(A) \cup \bigcup_{a \in A}(a N-\mathcal{E}(a-A))\right) .
$$

We have been unable to find exactly this result in the literature. It would be good to obtain an upper bound on $N_{A}$, presumably in terms of the geometry of the convex hull of $A$.

In Theorem 1, when $A \subset \mathbb{N}^{1}$, the sets $\mathcal{E}(A)$ are finite, which can be viewed as a finite union of 0 dimensional objects. In the two dimensional example

$$
A=\{(0,0),(2,0),(0,3),(1,1)\},
$$

we find that $\mathcal{E}(A)$ in infinite, explicitly

$$
\begin{aligned}
\mathcal{E}(A)= & \{(0,1),(1,0),(1,2)\}+\mathcal{P}(\{(0,0),(2,0)\}) \\
\cup & \{(0,1),(0,2),(1,0),(1,2),(2,1),(3,0)\}+\mathcal{P}(\{(0,0),(0,3)\}),
\end{aligned}
$$

the union of nine one-dimensional objects. More generally we prove the following:

Theorem 3 Let $0 \in A \subset \mathbb{Z}^{n}$ such that $A$ spans $\mathbb{Z}^{n}$ as a vector space over $\mathbb{Z}$. Then $\mathcal{E}(A)$ is a finite union of sets of the form

$$
\left\{v+\sum_{b \in B} m_{b} b: m_{b} \in \mathbb{Z}_{\geq 0}\right\}=v+\mathcal{P}(B \cup\{0\})
$$

where $v \in C_{A} \cap \mathbb{Z}_{\geq 0}^{n}$, with $B \subset A$ contains $\leq n-1$ elements, and the vectors in $B-0$ are linearly independent.

We deduce from Theorem 3 that

$$
\# \mathcal{E}_{N}(A)=O\left(N^{n-1}\right) .
$$

Theorem $\left[3\right.$ also implies that there is a bound $B_{A}$ such that every element of $C_{A} \cap \mathbb{Z}^{n}$ which is further than a distance $B_{A}$ from its boundary, is an element of $\mathcal{P}(A)$ (and so not in $\mathcal{E}(A)$ ).

The most remarkable result in this area is the 1992 theorem of Khovanskii [Kh92, Corollary 1] who proved that \#NA is a polynomial of degree $n$ in $N$ for $N$ sufficiently large, where the leading coefficient is $\operatorname{Vol}(H(A))$. His extraordinary proof proceeds by constructing a finitely-generated graded module $M_{1}, M_{2}, \ldots$ over $\mathbb{C}\left[t_{1}, \ldots, t_{k}\right]$ with $k=\# A$, where each $M_{N}$ is a vector space over $\mathbb{C}$ of dimension $|N A|$. One then deduces that $|N A|=\operatorname{dim}_{\mathbb{C}} M_{N}$ is a polynomial in $N$, for $N$ sufficiently large, by a theorem of Hilbert. Nathanson [Nat00] showed that this can generalized to sums $N_{1} A_{1}+\cdots+N_{k} A_{K}$ when all the $N_{i}$ are sufficiently large. This was all reproved by Nathanson and Ruzsa [NaRu02 using elementary, combinatorial ideas (using several ideas in common with us). Moreover it can also be deduced from Theorems 2 and 3 .

In section 2 we look at the case where $A$ has three elements, showing that the result holds for all $N \geq 1$. This easier case introduces some of the ideas we will need later. In section 3 we prove Theorem 1. Obtaining the bound $N \geq 2 b-2$ is not especially difficult, but improving this to $N \geq 2\left[\frac{b}{2}\right]$ becomes complicated and so we build up to it 
in a number of steps. In section 4 we begin the study of a natural higher dimensional analog. The introduction of even one new dimension creates significant complications, as the exceptional set $\mathcal{E}(A)$ is no longer necessarily finite. In the next subsection we indicate how one begins to attack these questions.

\subsection{Representing most elements of $\mathbb{Z}_{\geq 0}^{n}$. If $A=\{0,3,5\}$ one can represent}

$$
8=1 \times 3+1 \times 5, \quad 9=3 \times 3 \text { and } 10=2 \times 5
$$

and then every integer $n \geq 11$ is represented by adding a positive multiple of 3 to one of these representations, depending on whether $n \equiv 2,0$ or $1 \bmod 3$, respectively. In effect we are find representatives $r_{1}=10, r_{2}=8, r_{3}=9$ of $\mathbb{Z} / 3 \mathbb{Z}$ that belong to $\mathcal{P}(A)$, and then $\mathbb{Z}_{\geq 8}=\left\{r_{1}, r_{2}, r_{3}\right\}+3 \mathbb{Z}_{\geq 0} \subset \mathcal{P}(A)$, which implies that $\mathcal{E}(A) \subset\{0, \ldots, 7\}$.

We can generalize this to arbitrary finite $A \subset \mathbb{Z}_{>0}$ with $\operatorname{gcd}(a: a \in A)=1$, as follows: Let $b \geq 1$ be the largest element of $A$ (with 0 the smallest). Since $\operatorname{gcd}(a: a \in$ $A)=1$ there exist integers $m_{a}$, some positive, some negative, for which $\sum_{a \in A} m_{a} a=1$. Let $m:=\max _{a \in A}\left(-m_{a}\right)$ and $N:=b m \sum_{a \in A} a$, so that

$$
r_{k}:=N+k=\sum_{a \in A}\left(b m+k m_{a}\right) a \in \mathcal{P}(A) \text { for } 1 \leq k \leq b
$$

$\left(\right.$ as each $\left.b m+k m_{a} \geq b m-k m \geq 0\right)$ and $r_{k} \equiv k \bmod b$. But then

$$
\mathbb{Z}_{>N}=N+b \mathbb{Z}_{\geq 1}=N+\{1, \ldots, b\}+b \mathbb{Z}_{\geq 0}=\left\{r_{1}, \ldots, r_{b}\right\}+b \mathbb{Z}_{\geq 0} \subset \mathcal{P}(A),
$$

which implies that $\mathcal{E}(A) \subset\{0, \ldots, N\}$.

We can proceed similarly in $\mathbb{Z}_{\geq 0}^{n}$ with $n>1$, most easily when $C_{A}$ is generated by a set $B$ containing exactly $n$ non-zero elements (for example, $B:=\{(0,0),(2,0),(0,3)\} \subset$ $A$, in the example from (1) ). Let $\Lambda_{B}$ be the lattice of integer linear combinations of elements of $B$. We need to find $R \subset \mathcal{P}(A)$, a set of representatives of $\mathbb{Z}^{n} / \Lambda_{B}$, and then $\left(R+C_{B}\right) \cap \mathbb{Z}^{n} \subset \mathcal{P}(A)$. In the example (11) we can easily represent $\left\{(m, n) \in \mathbb{Z}^{2}: 4 \leq\right.$ $m \leq 5,3 \leq n \leq 5\}$. Therefore if $(r, s) \in \mathcal{E}(A)$ then either $0 \leq r \leq 3$ or $0 \leq s \leq 2$, and so we see that $\mathcal{E}(A)$ is a subset of a finite set of translates of one-dimensional objects.

\section{Classical postage stamp problem with at most $N$ stamps}

It is worth pointing out explicitly that if, for given coprime integers $0<a<b$, we have $n \in N\{0, a, b\}$ so that $n=a x+b y$ with $x+y \leq N$ then

$$
(N-x-y) \times b+x \times(b-a)=b N-n
$$

so that $b N-n \in N\{0, b-a, b\}$.

Theorem 4 (Postage Stamp with at most $N$ stamps) Let $0<a<b$ be coprime integers and $A=\{0, a, b\}$. If $N \geq 1$ then

$$
N A=\{0, \ldots, b N\} \backslash(\mathcal{E}(A) \cup(b N-\mathcal{E}(b-A))) .
$$

In other words, $N A$ contains all the integers in $[0, b N]$, except a few unavoidable exceptions near to the endpoints of the interval.

\footnotetext{
${ }^{5}$ In this displayed equation, and throughout, we write " $r \times a$ " to mean $r$ copies of the integer $a$.
} 
Proof. Suppose that $n \in\{0, \ldots, b N\}, n \notin \mathcal{E}(A)$ and $b N-n \notin \mathcal{E}(b-A)$, so that there exist $r, s, r^{\prime}, s^{\prime} \in \mathbb{N}$ such that

$$
r a+s b=n
$$

and

$$
r^{\prime}(b-a)+s^{\prime} b=b N-n .
$$

We may assume $0 \leq r, r^{\prime} \leq b-1$, as we may replace $r$ with $r-b$ and $s$ with $s+a$, and $r^{\prime}$ with $r^{\prime}-b$ and $s^{\prime}$ with $s^{\prime}+b-a$. Now reducing (3) and (44) modulo $b$, we have

$$
r a \equiv n(\bmod b), \quad-r^{\prime} a \equiv-n(\bmod b) .
$$

Since $(a, b)=1$, we deduce $r \equiv r^{\prime}(\bmod b)$. Therefore $r=r^{\prime}$ as $\left|r-r^{\prime}\right|<b$, and so adding (3) and (41) we find

$$
r b+s b+s^{\prime} b=b N .
$$

This implies that $r+s+s^{\prime}=N$ and so $r+s \leq N$ which gives $n \in N A$, as desired.

\section{Arbitrary postage problem with at most $N$ Stamps}

\subsection{Sets with three or more elements. Let}

$$
A=\left\{0=a_{1}<a_{2}<\ldots<a_{k}=b\right\} \subset \mathbb{Z},
$$

with $\left(a_{1}, \ldots, a_{k}\right)=1$. In general we have $n \in N A$ if and only if $N b-n \in N(b-A)$, since

$$
n=\sum_{i=1}^{k} m_{i} a_{i} \text { if and only if } N b-n=\sum_{i=1}^{k} m_{i}\left(b-a_{i}\right)
$$

where we select $m_{1}$ so that $\sum_{i=1}^{k} m_{i}=N$. For $0 \leq a \leq b-1$ define

$$
n_{a, A}:=\min \{n \geq 0: n \equiv a(\bmod b) \text { and } n \in \mathcal{P}(A)\}
$$

and

$$
N_{a, A}:=\min \left\{N \geq 0: n_{a, A} \in N A\right\}
$$

We always have $n_{0, A}=0$ and $N_{0, A}=0$. Neither 0 nor $b$ can be a term in the sum for $n_{a, A}$ else we can remove it and contradict the definition of $n_{a, A}$. But this implies that $n_{a, A} \leq N_{a, A} \cdot \max _{c \in A: c<b} c \leq(b-1) N_{a, A}$.

Lemma 1 If $n \equiv a(\bmod b)$ then $n \in \mathcal{P}(A)$ if and only if $n \geq n_{a, A}$.

Proof. If $n<n_{a, A}$ then $n \notin \mathcal{P}(A)$ by the definition of $n_{a, A}$. Write $n_{a, A}=\sum_{c \in A} n_{c} c$ where each $n_{c} \geq 0$. If $n \equiv a(\bmod b)$ and $n \geq n_{a, A}$ then $n=n_{a, A}+r b$ for some integer $r \geq 0$ and so $n=\sum_{c \in A, c \neq b} n_{c} c\left(n_{b}+r\right) b \in \mathcal{P}(A)$.

We deduce that

$$
\mathcal{E}(A)=\bigcup_{a=1}^{b-1}\left\{1 \leq n<n_{a, A}: n \equiv a(\bmod b)\right\}
$$

We also have the following: 
Corollary 1 Suppose that $0 \leq n \leq b N$ and $n \equiv a(\bmod b)$. Then

$$
n \notin \mathcal{E}(A) \cup(N b-\mathcal{E}(b-A)) \text { if and only if } n_{a, A} \leq n \leq b N-n_{b-a, b-A} \text {. }
$$

Thus there are such integers $n$ if and only if $N \geq N_{a, A}^{*}:=\frac{1}{b}\left(n_{a, A}+n_{b-a, b-A}\right)$.

Lemma 2 Suppose that $N_{0} \geq N_{a, A}^{*}$. Assume that if $0 \leq n \leq b N_{0}$ with $n \equiv a(\bmod b)$, and $n \notin \mathcal{E}(A) \cup\left(N_{0} b-\mathcal{E}(b-A)\right)$ then $n \in N_{0} A$. Then for any integer $N \geq N_{0}$ we have $n \in N A$ whenever $0 \leq n \leq b N$ with $n \equiv a(\bmod b)$, and $n \notin \mathcal{E}(A) \cup(N b-\mathcal{E}(b-A))$.

Proof. By induction. By hypothesis it holds for $N=N_{0}$. Suppose it holds for some $N \geq N_{0}$. If $n \equiv a(\bmod b)$ with $a \leq n \leq b(N+1)-n_{b-a, b-A}$ then either $a \leq n \leq$ $b N-n_{b-a, b-A}$ so that $n \in N A \subset(N+1) A$, or $n=b+\left(b N-n_{b-a, b-A}\right) \in b+N A \subset$ $(N+1) A$.

$$
\begin{aligned}
& \text { If } n_{a, A}=a_{1}+\cdots+a_{N} \text { where } N=N_{a, A} \text { then } \\
& \qquad b N_{a, A}-n_{a, A}=\left(b-a_{1}\right)+\cdots+\left(b-a_{N}\right) \geq n_{b-a, b-A},
\end{aligned}
$$

by definition. Therefore

$$
N_{a, A} \geq \frac{1}{b}\left(n_{a, A}+n_{b-a, b-A}\right)=N_{a, A}^{*},
$$

and the analogous argument implies that $N_{b-a, b-A} \geq N_{a, A}^{*}$.

Corollary 2 Given a set $A$, fix $a(\bmod b)$. The statement "For all integers $N \geq 1$, for all integers $n \in[0, N b]$ with $n \equiv a(\bmod b)$ we have $n \in N A$ if and only if $n \notin \mathcal{E}(A) \cup(N b-\mathcal{E}(b-A))$ " holds true if and only if $N_{a, A}=N_{a, A}^{*}$.

Proof. There are no such integers $n$ if $N<N_{a, A}^{*}$ by Corollary 1, so the statement is true. If the statement is true for $N=N_{a, A}^{*}$ then it holds for all $n \geq N_{a, A}^{*}$ by Lemma 2 , Finally for $N=N_{a, A}^{*}$, the statement claims (only) that $n_{a, A} \in N A$. This happens if and only if $N=N_{a, A}^{*} \geq N_{a, A}$. The result follows since we just proved that $N_{a, A} \geq N_{a, A}^{*}$.

In fact one can re-run the proof on $b N-a$ to see that if $N_{a, A}=N_{a, A}^{*}$ then $N_{b-a, b-A}=$ $N_{a, A}^{*}$. Suppose $A$ has just three elements, say $A=\{0, c, b\}$ with $(c, b)=1$. For any non-zero $a(\bmod b)$ we have an integer $r, 1 \leq r \leq b-1$ with $a \equiv c r(\bmod b)$, and one can easily show that $n_{a, A}=c r$ while $N_{a, A}=r$. Now $b-A=\{0, b-c, b\}$ so that $n_{b-a, b-A}=$ $(b-c) r$ while $N_{b-a, b-A}=r$. Therefore $N_{a, A}=N_{b-a, b-A}=N_{a, A}^{*}=\frac{1}{b}\left(n_{a, A}+n_{b-a, b-A}\right)$ for every $a$, and so we recover Theorem 4 from Corollary 2 ,

However Theorem 1 does not hold for all $N \geq 1$ for some sets $A$ of size 4 . For example, if $A=\{0,1, b-1, b\}$ then $b-A=A$. We have $n_{a, A}=a$ for $1 \leq a \leq b-1$, and so $N_{a, A}^{*}=1$, but $N_{a, A}=a$ for $1 \leq a \leq b-2$, and so Theorem 1 does not hold for all $N \geq 1$ by Corollary 2. In fact since $N_{b-2, b}=b-2>N_{b-2, b}^{*}=1$, if the statement "if $n \leq N b$ and $n \notin \mathcal{E}(A) \cup(N b-\mathcal{E}(b-A))$ then $n \in N A$ " is true then $N \geq b-2$.

It would be interesting to have a simple criterion for the set $A$ to have the property that $N_{a, A}=N_{a, A}^{*}$ for all $a(\bmod b)$ (so that Corollary 2 takes effect). Certainly many sets $A$ do not have this property; For example if there exists an integer $a, 1 \leq a \leq b-1$ such that $a \notin A$ but $a, b+a \in 2 A$, then $n_{a, A}=a, n_{b-a, b-A}=b-a$, so that $N_{a, A}=2$ and $N_{a, A}^{*}=1$. 
3.2. Proving a "sufficiently large" result. We begin getting bounds by proving the following.

Proposition 1 Fix $0 \leq a \leq b-1$ and suppose $N \geq N_{a, A}+N_{b-a, b-A}$. If $0 \leq n \leq N b$ with $n \equiv a(\bmod b)$ and $n \notin \mathcal{E}(A) \cup(N b-\mathcal{E}(b-A))$ then $n \in N A$.

Corollary 3 If $0 \leq n \leq N b$ and $n \notin \mathcal{E}(A) \cup(N b-\mathcal{E}(b-A))$ then $n \in N A$, whenever $N \geq \max _{1 \leq a \leq b-1} N_{a, A}+N_{b-a, b-A}$.

To prove Proposition 1, we need the following.

Proposition 2 Fix $1 \leq a \leq b-1$. If $n \leq\left(N-N_{a, A}\right) b$ with $n \equiv a(\bmod b)$ and $n \notin \mathcal{E}(A)$ then $n \in N A$.

Proof. If $n \notin \mathcal{E}(A)$ then $n \geq n_{a, A}$ by the definition of $n_{a, A}$. Therefore $n=n_{a, A}+k b$ where $0 \leq k b \leq n \leq\left(N-N_{a, A}\right) b$, so that $0 \leq k \leq N-N_{a, A}$ and $k b \in\left(N-N_{a, A}\right) A$. Now $n_{a, A} \in N_{a, A} A$ and so $n=n_{a, A}+k b \in N_{a, A} A+\left(N-N_{a, A}\right) A=N A$.

Proof of Proposition 1. This is trivial for $a=0$. Otherwise, by hypothesis $n \notin \mathcal{E}(A)$ and $b N-n \notin \mathcal{E}(b-A)$. Moreover either $n \leq\left(N-N_{a, A}\right) b$ or $b N-n \leq\left(N-N_{b-a, b-A}\right) b$, else

$b N=n+(b N-n)>\left(N-N_{a, A}\right) b+\left(N-N_{b-a, b-A}\right) b=\left(2 N-N_{a, A} A-N_{b-a, b-A}\right) b \geq N b$,

which is impossible. Therefore Proposition 1 either follows by applying Proposition 2 to $A$, or by applying Proposition 2 to $b-A$ to obtain $N b-n \in N(b-A)$ which implies $n \in N A$.

It remains to bound $N_{a, A}$. We start with the following.

Lemma 3 We have $N_{a, A} \leq b-1$. If $A=\{0,1, b\}$ then $N_{a, A}=b-1$.

Proof. Suppose that $n_{a, A}=a_{1}+a_{2}+\cdots+a_{r}$ with each $a_{i} \in A$, and $r$ minimal. We have $r<b$ else two of $0, a_{1}, a_{1}+a_{2}, \ldots, a_{1}+\cdots+a_{b}$ are congruent mod $b$ by the pigeonhole principle, so their difference, which is a subsum of the $a_{i}$ 's is $\equiv 0(\bmod b)$. If these $a_{i}$ 's are removed from the sum then we obtain a smaller element of $\mathcal{P}(A)$ that is $\equiv a(\bmod b)$, contradicting the definition of $n_{a, A}$. We deduce that $N_{A} \leq b-1$. If $A=\{0,1, b\}$ then $b-1 \notin(b-2) A$ and so $N_{A} \geq b-1$.

Corollary 4 Suppose that $N \geq 2 b-2$. If $n \leq N b$ and $n \notin \mathcal{E}(A) \cup(N b-\mathcal{E}(b-A))$ then $n \in N A$.

Proof. Insert the bounds $N_{a, A}, N_{b-a, b-A} \leq b-1$ from Lemma 3 into Corollary 3 .

3.3. The proof of Theorem 1. With more effort we now prove Theorem 1, improving upon Corollary 4 by a factor of 2 , and getting close to the best possible bound $b-2$ (which, as we have seen, is as good as can be attained when $A=\{0,1, b-1, b\}$ ). One cannot obtain a better consequence of Corollary 3 since we have the following examples:

$$
\text { If } A=\{0,1, b-1, b\} \text { then } N_{\left[\frac{b}{2}\right], A}+N_{b-\left[\frac{b}{2}\right], b-A}=2\left[\frac{b}{2}\right] \text {. }
$$


If $A=\{0,1,2, b\}$ with $b$ even then $N_{b-1, A}+N_{1, b-A}=b$. This is a particularly interesting case as one can verify that one has "If $n \leq N b$ and $n \notin \mathcal{E}(A) \cup(N b-\mathcal{E}(b-A))$ then $n \in N A$ " for all $N \geq 1$.

We can apply Corollary 3 to obtain Theorem 1 provided $N_{a, A}, N_{b-a, b-A} \leq\left[\frac{b}{2}\right]$ for each $a$. Therefore we need to classify those $A$ for which $N_{a, A}>\frac{b}{2}$

Let $(t)_{b}$ is the least non-negative residue of $t(\bmod b)$.

Suppose that $1 \leq a \leq b-1$, and write $n_{a}=n_{a, A}=a_{1}+\cdots+a_{m}$ where $m=N_{a, A}$ is minimal. No subsum of $a_{1}+\cdots+a_{m}$ can sum to $\equiv 0(\bmod b)$ else we remove this subsum from the sum to get a smaller sum of elements of $A$ which is $\equiv a(\bmod b)$, contradicting the definition of $n_{a}$. Also the complete sum cannot be $\equiv 0(\bmod b)$ else $a=0$ and $m=0$. Let $k=m+1$ and $a_{k}=-\left(a_{1}+\cdots+a_{m}\right)$, so that $a_{1}+\cdots+a_{k} \equiv 0(\bmod b)$ and no proper subsum is $0(\bmod b)$; we call this a minimal zero-sum. The Savchev-Chen structure theorem [SaChe07] states that if $k \geq\left[\frac{b}{2}\right]+2$ then $a_{1}+\cdots+a_{k} \equiv 0(\bmod b)$ is a minimal zero-sum if and only if there is a reduced residue $w(\bmod b)$ and positive integers $c_{1}, \ldots, c_{k}$ such that $\sum_{j} c_{j}=b$ and $a_{j} \equiv w c_{j}(\bmod b)$ for all $j$.

Theorem 3.1 If $N_{a, A}>\frac{b}{2}$ then $n_{a, A}$ is the sum of $N_{a, A}$ copies of some integer $h, 1 \leq$ $h \leq b-1$ with $(h, b)=1$. Moreover if $k \in A$ with $\ell \neq h$ then $(k / h)_{b} \geq N_{a, A}+1$.

Proof. Above we have $k=m+1=N_{a, A}+1 \geq\left[\frac{b}{2}\right]+2$ so we can apply the Savchev-Chen structure theorem. Some $c_{j}$ with $j \leq m$ must equal 1 else $b=\sum_{j=1}^{m} c_{j} \geq 2 m>b$, a contradiction. Hence $h \in A$ where $h=(w)_{b}$. Let $n:=\#\left\{j \in[1, m]: c_{j}=1\right\}=\#\{j \in$ $\left.[1, m]: a_{j}=h\right\} \geq 1$.

If $(\ell h)_{b} \in A$ where $1 \leq \ell<b$ then $n \leq \ell-1$ else we can remove $\ell$ copies of $h$ from the original sum for $n_{a, A}$ and replace them by one copy of $(\ell h)_{b}$. If $(\ell h)_{b}<\ell h$ then this makes the sum smaller, contradicting the definition of $n_{a}$. Otherwise this makes the number of summands smaller contradicting the definition of $N_{a, A}$.

Therefore if $k$ is the smallest $c_{j}$-value $>1$, with $1 \leq j \leq m$, then $(k h)_{b} \in A$ so that $k \geq n+1$, and so

$$
b-1 \geq \sum_{j=1}^{m} c_{j} \geq n \times 1+(m-n) \times k=m+(m-n)(k-1) \geq m+(m-n) n .
$$

If $1 \leq n \leq m-1$ then this gives $b-1 \geq m+(m-1)>b-1$, a contradiction. Hence $n=m$; that is, $n_{a}=h+h+\cdots+h$. Therefore $h m \equiv a(\bmod b)$. Moreover if $(\ell h)_{b} \in A$ with $\ell \neq 1$ then $\ell \geq n+1=m+1$.

We now give a more precise version of the argument in Proposition 2 ,

Proposition 3 Fix $0 \leq a \leq b-1$ and suppose $N \geq \max \left\{N_{a, A}, N_{b-a, b-A}\right\}$. For all $0 \leq n \leq N b$ with $n \equiv a(\bmod b)$ and $n \notin \mathcal{E}(A) \cup(N b-\mathcal{E}(b-A))$ we have that $n \in N A$, except perhaps if $n=n_{a, A}+j b$ where

$$
N-N_{a, A}<j<N_{b-a, b-A}-\frac{1}{b}\left(n_{a, A}+n_{b-a, b-A}\right) .
$$

Proof. Since $n_{a, A} \in N_{a, A} A$, we have

$$
n_{a, A}+j b \in\left(N_{a, A}+j\right) A \in N A \text { whenever } 0 \leq j \leq N-N_{a, A} .
$$


The analogous statement for $b-A$ implies that

$$
b N_{b-a, b-A}-n_{b-a, b-A}+i b \in N A \text { whenever } 0 \leq i \leq N-N_{b-a, b-A} .
$$

Proof of Theorem 11. Suppose that $N \geq N_{0}:=2\left[\frac{b}{2}\right] \geq b-1$. We will prove the result now for $N=N_{0}$; the result for all $N \geq N_{0}$ follows from Lemma 2 .

If $N_{a, A}, N_{b-a, b-A} \leq\left[\frac{b}{2}\right]$ then the result follows from Proposition 1. Hence we may assume that $N_{a, A}>\left[\frac{b}{2}\right]$ (if necessary changing $A$ for $b-A$ ).

Theorem 3.1 implies there exists an integer $h, 1 \leq h \leq b-1$ with $(h, b)=1$ such that $n_{a, A}=N_{a, A} \times h$. We already proved the result when $A$ has three elements, so we may now assume it has a fourth, say $\{0, h, \ell, b\} \subset A$.

Let $\mathcal{B}=\{0, h, \ell\} \subset \mathbb{Z} / b \mathbb{Z}$. Since $\mathcal{B}$ is not contained in any proper subgroup of $\mathbb{Z} / b \mathbb{Z}$ $($ as $(h, b)=1)$, Kneser's theorem implies that $|k \mathcal{B}| \geq 2 k+1$.

For $N_{0}-N_{a, A} \leq k \leq \frac{b-1}{2}$, let $S:=2 k-b+N_{a, A}+1$ so that there are $b-2 k$ elements in $\left\{S h,(S+1) h, \ldots, N_{a, A} h\right\}$. By the pigeonhole principle, $s h \in k \mathcal{B}$ for some $s, S \leq s \leq N_{a, A}$ and therefore $s h+t b=a_{1}+\cdots+a_{k}$ where each $a_{i} \in A$, for some integer $t$. Now $t \geq 0$ else we can replace $s h$ by $a_{1}+\cdots+a_{k}$ contradicting the definition of $n_{a, A}$. On the other hand, $t b<s h+t b=a_{1}+\cdots+a_{k} \leq k(b-1)$ and so $t \leq k$. Therefore

$$
\begin{aligned}
n_{a, A}+k b & =\left(N_{a, A}-s\right) h+\left(a_{1}+\cdots+a_{k}\right)+(k-t) b \in\left(N_{a, A}-s+2 k-t\right) A \\
& \subset\left(N_{a, A}-S+2 k-t\right) A=(b-1-t) A \subset N_{0} A .
\end{aligned}
$$

We have filled in the range (5) for all $j \leq \frac{b-1}{2}$, which gives the whole of (5) if $N_{b-a, b-A} \leq$ $\left[\frac{b}{2}\right]$. Therefore we may now assume that $N_{b-a, b-A}>\left[\frac{b}{2}\right]$.

Since $N_{b-a, b-A}>\left[\frac{b}{2}\right]$ we may now rerun the argument above and obtain that

$$
n_{b-a, b-A}+k b \in N_{0}(b-A) \text { for all } k \leq \frac{b-1}{2},
$$

and therefore if $n_{a, A}+j b \notin \mathcal{E}(b-A)$ then

since

$$
n_{a, A}+j b \in N_{0} A \text { for all } j \geq \frac{b-1}{2},
$$

$$
N_{0}-\frac{b-1}{2}-\frac{n_{a, A}+n_{b-a, b-A}}{b} \leq b-\frac{b-1}{2}-1=\frac{b-1}{2} .
$$

\section{Higher Dimensional POStage StAmp PROBLEM}

Let $A=\left\{a_{1}, \ldots, a_{k}\right\} \subset \mathbb{Z}^{n}$ be a finite set of vectors with $k \geq n+2$. After translating $A$, we assume that $0 \in A$ so that

$$
0 \in A \subset 2 A \subset \cdots .
$$

We are interested in what elements are in $N A$. Assume that

$$
\Lambda_{A}:=\langle A\rangle_{\mathbb{Z}}=\mathbb{Z}^{n} .
$$

It is evident from the definitions that

$$
N A \subset N H(A) \cap \mathcal{P}(A)=\left(N H(A) \cap \mathbb{Z}^{n}\right) \backslash \mathcal{E}(A)
$$


Let $b \in A$ and suppose that $x \in N A$ so that $x=\sum_{a \in A} c_{a} a$ where the $c_{a}$ are nonnegative integers that sum to $N$. Therefore $N b-x=N b-\sum_{a \in A} c_{a} a=\sum_{a \in A} c_{a}(b-a) \in$ $N(b-A) \subset \mathcal{P}(b-A)$. This implies that $N b-x \notin \mathcal{E}(b-A)$, and so $x \notin N b-\mathcal{E}(b-A)$. Therefore

$$
N A \subset\left(N H(A) \cap \mathbb{Z}^{n}\right) \backslash \mathcal{E}_{N}(A)
$$

where

$$
\mathcal{E}_{N}(A):=N H(A) \cap\left(\mathcal{E}(A) \cup \bigcup_{a \in A}(a N-\mathcal{E}(a-A))\right) .
$$

In Theorem 2 we will show that this is an equality for large $N$. We use two classical lemmas to prove this, and include their short proofs.

\subsection{Two classical lemmas.}

Lemma 4 (Carathéodory's theorem) Assume that $0 \in A$ and $A-A$ spans $\mathbb{R}^{n}$. If $v \in N H(A)$ then there exists a subset $B \subset A$ which contains $n+1$ elements, such that $B-B$ is a spanning set for $\mathbb{R}^{n}$, for which $v \in N H(B)$.

Note that the condition $B-B$ spans $\mathbb{R}^{n}$ is equivalent to the condition that $B$ is not contained in any hyperplane. In two dimensions, Lemma 4 asserts that each point of a polygon lies in a triangle (which depends on that point) formed by 3 of the vertices.

Proof. Since $v \in N H(A)$ we can write

$$
v=\sum_{a \in A} c_{a} a \in N H(A), \text { with } 0 \leq \sum_{a \in A} c_{a} \leq N,
$$

where each $c_{a} \geq 0$. We select the representation that minimizes $\# B$ where

$$
B=\left\{a: c_{a}>0\right\}
$$

Select any $b_{0} \in B$. We now show that the vectors $b-b_{0}, b \in B, b \neq b_{0}$ are linearly independent over $\mathbb{R}$. If not we can write

$$
\sum_{b \in B \backslash\left\{b_{0}\right\}} e_{b}\left(b-b_{0}\right)=0,
$$

where the $e_{b}$ are not all 0 . Let $e_{b_{0}}=-\sum_{b} e_{b}$ so that $\sum_{b \in B} e_{b} b=0$ and $\sum_{b \in B} e_{b}=0$, and at least one $e_{b}$ is positive. Now let

$$
m=\min _{b: e_{b}>0} c_{b} / e_{b},
$$

where $c_{\beta}=m e_{\beta}$ with $\beta \in B$. Then $v=\sum_{b \in B}\left(c_{b}-m e_{b}\right) b$ where each $c_{b}-m e_{b} \geq 0$ with $\sum_{b \in B}\left(c_{b}-m e_{b}\right)=\sum_{b \in B} c_{b}-m \sum_{b \in B} e_{b}=\sum_{b \in B} c_{b} \in[0, N]$. However the coefficient $c_{\beta}-m e_{\beta}=0$ and this contradicts the minimality of $\# B$.

Since the vectors $b-b_{0}, b \in B, b \neq b_{0}$ are linearly independent, we can add new elements of $A$ to the set $B$ until we have $n+1$ elements, and then we obtain the result claimed.

For $u=\left(u_{1}, \ldots, u_{n}\right), v=\left(v_{1}, \ldots, v_{n}\right) \in \mathbb{Z}_{\geq 0}^{n}$, we write $u \leq v$ if $u_{i} \leq v_{i}$ for each $i=1, \ldots, n$. The following is a classical lemma in additive combinatorics: 6

\footnotetext{
${ }^{6}$ Formerly known as "additive number theory".
} 
Lemma 5 (Mann's lemma) Let $S \subset \mathbb{Z}_{\geq 0}^{n}$. There is a finite subset $T \subset S$ such that for all $s \in S$ there exists $t \in T$ for which $t \leq s$.

Proof. We prove by induction on $n \geq 1$. For convenience we will write $T \leq S$, if for all $s \in S$ there exists $t \in T$ for which $t \leq s$. For $n=1$ let $T=\{t\}$ where $t$ is the smallest integer in $S$. For $n>1$, select any element $\left(s_{1}, \ldots, s_{n}\right) \in S$. Define $S_{j, r}:=\left\{\left(u_{1}, \ldots, u_{n}\right) \in S: u_{j}=r\right\}$ for each $j=1, \cdots, n$ and $0 \leq r<s_{j}$. Let $\phi_{j}\left(\left(u_{1}, \ldots, u_{n}\right)\right)=\left(u_{1}, \cdots, u_{j-1}, u_{j+1}, \cdots, u_{n}\right)$. The set $\phi_{j}\left(S_{j, r}\right) \subset \mathbb{Z}_{>0}^{n-1}$ and so, by the induction hypothesis, there exists a finite subset $T_{j, r} \subset S_{j, r}$ such that $\bar{\phi}_{j}\left(T_{j, r}\right) \leq \phi_{j}\left(S_{j, r}\right)$, which implies that $T_{j, r} \leq S_{j, r}$ as their $j$ th co-ordinates are the same. Now let

$$
T=\left\{\left(s_{1}, \ldots, s_{n}\right)\right\} \bigcup_{j=1}^{n} \bigcup_{r=0}^{s_{j}-1} T_{j, r},
$$

which is a finite union of finite sets, and so finite. If $s \in S$ then either $\left(s_{1}, \ldots, s_{n}\right) \leq s$, or $s \in S_{j, r}$ for some $j, 1 \leq j \leq n$, and some $r, 0 \leq r<s_{j}$. Hence $T \leq S$.

Lemma 6 (Mann's lemma, revisited) Let $S \subset \mathbb{Z}_{\geq 0}^{n}$ with the property that if $s \in S$ then $s+\mathbb{Z}_{\geq 0}^{n} \in S$. Then $E:=\mathbb{Z}_{\geq 0}^{n} \backslash S$ is a finite union of sets of the form: For some $I \subset\{1, \ldots, n\}$

$$
\left\{\left(x_{1}, \ldots, x_{n}\right): x_{i} \in \mathbb{Z}_{\geq 0} \text { for each } i \in I\right\} \text { with } x_{j} \text { fixed if } j \notin I .
$$

Proof. By induction on $n \geq 1$. In 1-dimension, $S$ is either empty so that $E=\mathbb{Z}_{\geq 0}$, or $S$ has some minimum element $s$, in which case $E$ is the finite set of elements $0,1, \ldots, s-1$.

If $n>1$ then in $n$-dimensions either $S$ is empty so that $E=\mathbb{Z}_{\geq 0}^{n}$ or $S$ contains some element $\left(s_{1}, \ldots, s_{n}\right)$. Therefore if $\left(x_{1}, \ldots, x_{n}\right) \in E$ there must exist some $k$ with $x_{k} \in\left\{0,1, \ldots, s_{k}-1\right\}$. For each such $k, x_{k}$ we apply the result to $S_{x_{k}}:=\left\{\left(u_{1}, \ldots, u_{n}\right) \in\right.$ $\left.S: u_{k}=x_{k}\right\}$, which is $n-1$ dimensional.

4.2. The proof of Theorem 2 , For any $v \in \mathcal{P}(A)$ define

$$
\mu_{A}(v):=\min \left\{\sum_{a \in A} n_{a}: v=\sum_{a \in A} n_{a} a, \text { each } n_{a} \in \mathbb{N}\right\},
$$

and $\mu_{A}(V):=\max _{v \in V} \mu_{A}(v)$ for any $V \subset \mathcal{P}(A)$. By definition, $V \subset N A$ if and only if $N \geq \mu_{A}(V)$.

The heart of the proof of Theorem 2 is contained in the following result.

Proposition 4 Let $0 \in B \subset A \subset \mathbb{Z}^{n}$ where $\Lambda_{A}=\mathbb{Z}^{n}$, and $B^{*}=B \backslash\{0\}$ contains exactly $n$ elements, which span $\mathbb{R}^{n}$ (as a vector space over $\mathbb{R}$ ). There exists a finite subset $A^{+} \subset \mathcal{P}(A)$ such that if $v \in \mathcal{P}(A)$ then there is some $w=w(v) \in A^{+}$for which $v-w \in \mathcal{P}(B)$. (That is, $\mathcal{P}(A)=A^{+}+\mathcal{P}(B)$.) Let $N_{A, B}=\mu_{A}\left(A^{+}\right)$so that $A^{+} \subset N_{A, B} A$. If $N \geq N_{A, B}$ and $v \in\left(N-N_{A, B}\right) H(B) \cap \mathbb{Z}^{n}$ but $v \notin \mathcal{E}(A)$ then $v \in N A$.

Proof. The fundamental domain for the lattice $\Lambda_{B}:=\langle B\rangle_{\mathbb{Z}}$ is

$$
\mathbb{R}^{n} / \Lambda_{B} \cong \mathcal{F}(B):=\left\{\sum_{b \in B^{*}} c_{b} b: \text { Each } c_{b} \in[0,1)\right\}
$$


Since $\mathcal{F}(B)$ is bounded, we see that

$$
L:=\mathcal{F}(B) \cap \mathbb{Z}^{n}
$$

is finite. The sets $\ell+\Lambda_{B}$ partition $\mathbb{Z}^{n}$ as $\ell$ varies over $\ell \in L$. For each $\ell \in L$ we define

$$
A_{\ell}=\left(\ell+\Lambda_{B}\right) \cap \mathcal{P}(A),
$$

which partition $\mathcal{P}(A)$ into disjoint sets, so that $\mathcal{P}(A)=\bigcup_{\ell \in L} A_{\ell}$. Define $S_{\ell} \subset \mathbb{N}^{n}$ by

$$
A_{\ell}:=\left\{\ell+\sum_{b \in B^{*}} c_{b} b:\left(c_{1}, \ldots, c_{n}\right) \in S_{\ell}\right\} \subset C_{B} .
$$

By Mann's lemma (Lemma 5), there is a finite subset $T_{\ell} \subset S_{\ell}$ such that for each $s \in S_{\ell}$ there is a $t \in T_{\ell}$ satisfying $t \leq s$. We may assume that $T_{\ell}$ is minimal, and define

$$
A_{\ell}^{+}=\left\{\ell+\sum_{b \in B^{*}} c_{b} b:\left(c_{1}, \ldots, c_{n}\right) \in T_{\ell}\right\} \subset A_{\ell} .
$$

By definition, for any $v \in A_{\ell}$ there exists $w \in A_{\ell}^{+}$such that $v-w \in \mathcal{P}(B)$ (for we write $v=\ell+s \cdot B$ and let $w=\ell+t \cdot B$ where $t \leq s$, as above). That is, $A_{\ell}=A_{\ell}^{+}+\mathcal{P}(B)$.

Let $A^{+}=\cup_{\ell \in L} A_{\ell}^{+}$which is a finite union of finite sets, and so is finite, and $A^{+} \subset$ $\mathcal{P}(A)$. Moreover $\mathcal{P}(A)=\bigcup_{\ell \in L} A_{\ell}=\bigcup_{\ell \in L} A_{\ell}^{+}+\mathcal{P}(B)=A^{+}+\mathcal{P}(B)$ as claimed.

Now suppose that $v \in\left(N-N_{A, B}\right) H(B) \subset C_{B} \subset C_{A}$. Since the vectors in $B$ are linearly independent there is a unique representation $v=\sum_{b} v_{b} b$ as a linear combination of the elements of $B$, and has each $v_{b} \geq 0$ with $\sum_{b} v_{b} \leq N-N_{A, B}$.

Also suppose $v \in \mathbb{Z}^{n}$ but $v \notin \mathcal{E}(A)$ so that $v \in \mathcal{P}(A)$, as $v \in C_{A} \cap \mathbb{Z}^{n}$. Therefore there exists a unique $\ell \in L$ for which $v \in A_{\ell}$, and $w=w(v)=\sum_{b} w_{b} b \in A_{\ell}^{+}$for which each $0 \leq w_{b} \leq v_{b}$. Therefore $v-w=\sum_{b}\left(v_{b}-w_{b}\right) b \in U B$ where $U:=\sum_{b}\left(v_{b}-w_{b}\right) \leq$ $\sum_{b} v_{b} \leq N-N_{A, B}$ and so $v-w \in\left(N-N_{A, B}\right) B$. By definition, $w \in N_{A, B} A$, and so

$$
v=(v-w)+w \in\left(N-N_{A, B}\right) B+N_{A, B} A \subset\left(N-N_{A, B}\right) A+N_{A, B} A=N A .
$$

Proof of Theorem 2. For every subset $B \subset A$ which contains $n+1$ elements, such that $B-B$ is a spanning set for $\mathbb{R}^{n}$, define $N_{A, B}^{*}:=N_{A, B}+\sum_{b \in B, b \neq 0} N_{b-A, b-B}$, and let $N_{A}$ be the maximum of these $N_{A, B}^{*}$. If $N \geq N_{A}$ and $v \in N H(A)$ then $v \in N H(B)$ for some such set $B$, by Lemma 4 . If we also have $v \in \mathbb{Z}^{n}$ but

$$
v \notin \mathcal{E}(A) \cup \bigcup_{b \in B, b \neq 0}(N b-\mathcal{E}(b-A))
$$

then we can write $v=\sum_{b \in B} c_{b} b$ for real $c_{b} \geq 0$ with

$$
\sum_{b \in B} c_{b}=N \geq N_{A, B}+\sum_{b \in B, b \neq 0} N_{b-A, b-B}
$$

Therefore

- Either $c_{0} \geq N_{A, B}$ in which case

$$
v=\sum_{b \in B, b \neq 0} c_{b} b \in\left(N-c_{0}\right) H(B) \subset\left(N-N_{A, B}\right) H(B)
$$

as well as $v \in \mathbb{Z}^{n} \backslash \mathcal{E}(A)=\mathcal{P}(A)$, and so $v \in N A$ by Proposition 4, 
- Or there exists $\beta \in B, \beta \neq 0$ for which $c_{\beta} \geq N_{\beta-A, \beta-B}$ so that

$$
\beta N-v=\sum_{b \in B} c_{b}(\beta-b) \in\left(N-c_{\beta}\right) H(\beta-B) \subset\left(N-N_{\beta-A, \beta-B}\right) H(\beta-B) .
$$

Now $v, \beta \in \mathbb{Z}^{n}$ and so $\beta N-v \in \mathbb{Z}^{n}$. Also $v \notin \beta N-\mathcal{E}(\beta-A)$ by hypothesis, and so $\beta N-v \notin \mathcal{E}(\beta-A)$. Therefore $\beta N-v \in N(\beta-A)$ by Proposition 4 , giving that $v \in N A$.

\section{The STRUCTURE AND SIZE OF THE EXCEPTIONAL SET}

Proposition 5 Let $0 \in B \subset A \subset \mathbb{Z}^{n}$ where $\Lambda_{A}=\mathbb{Z}^{n}$, and $B^{*}=B \backslash\{0\}$ contains exactly $n$ elements, which span $\mathbb{R}^{n}$, so that $C_{B}=\left\{\sum_{b \in B^{*}} x_{b} b\right.$ : Each $\left.x_{b} \geq 0\right\}$. There exist $r_{b} \geq 0$ such that $\left\{\sum_{b \in B^{*}} x_{b} b:\right.$ Each $\left.x_{b} \geq r_{b}\right\} \cap \mathbb{Z}^{n} \subset \mathcal{P}(A)$.

We deduce that if $x:=\sum_{b \in B^{*}} x_{b} b \in\left(C_{B} \cap \mathbb{Z}^{n}\right) \cap \mathcal{E}(A)$ then $0 \leq x_{b}<r_{b}$ for some $b$. In other words $x$ is at a bounded distance from the boundary generated by $B \backslash\{b\}$. (Theorem 2 of SiTi03] gives a related result but is difficult to interpret in the language used here.)

Proof. We will use the notation of Proposition 4. The elements of $B^{*}$ are linearly independent so that $\beta:=\sum_{b \in B} b$ lies in the interior of $C_{B}$. Therefore if the integer $M$ is sufficiently large then $\gamma:=\beta+\frac{1}{M} \sum_{a \in A} a$ also lies in the interior of $C_{B}$.

Now as $A$ generates $\mathbb{Z}^{n}$ as a vector space over $\mathbb{Z}$, we know that for each $\ell \in L$ there exist integers $c_{\ell, a}$ such that $\ell=\sum_{a \in A} c_{\ell, a} a$. Let $c \geq 0$ be an integer $\geq \max _{\ell \in L, a \in A}\left(-c_{\ell, a}\right)$. The set $L^{\prime}=c M \gamma+L$ of $\mathbb{Z}^{n}$-points is a translate of $L$ that can be represented as

$$
c M \gamma+\sum_{a \in A} c_{\ell, a} a=c M \beta+\sum_{a \in A}\left(c+c_{\ell, a}\right) a \in \mathcal{P}(A) \text { for each } \ell \in L .
$$

The translation is by $c M \gamma \in C_{B}$ so $L^{\prime}=c M \gamma+L \subset C_{B}$; moreover $L^{\prime}$ gives a complete set of representatives of $\mathbb{R}^{n} / \Lambda_{B}$ and so every lattice point in $L^{\prime}+\mathcal{P}(B)$ belongs to $\mathcal{P}(A)$. We can re-phrase this as

$$
\left(c M \gamma+C_{B}\right) \cap \mathbb{Z}^{n} \subset \mathcal{P}(A) .
$$

Therefore if $c M \gamma=\sum_{b \in B^{*}} r_{b} b$ and $x:=\sum_{b \in B^{*}} x_{b} b \in \mathbb{Z}^{n}$, then $x \in \mathcal{P}(A)$ if each $x_{b} \geq r_{b}$.

Proof of Theorem 3. We again use Lemma 4 to focus on sets $B \subset A$ which contain $n+1$ elements, such that $B-B$ is a spanning set for $\mathbb{R}^{n}$. We translate $B$ so that $0 \in B$. As in the proof of Proposition 4, we fix $\ell \in L$ (which is a finite set). Proposition 5 shows that $S_{\ell}$ is non-empty. Lemma 6 yields the structure of $\mathbb{Z}_{\geq 0}^{n} \backslash S_{\ell}$, which is not all of $\mathbb{Z}_{\geq 0}^{n}$ as $S_{\ell}$ contains an element. This implies that the structure of $\left(\ell+\Lambda_{B}\right) \cap \mathcal{E}(A)$ is as claimed in Theorem 3 . The result follows as $\mathcal{E}(A)$ is a finite union of such sets.

\section{REFERENCES}

[Di90] J. Dixmier, Proof of a conjecture by Erdös and Graham concerning the problem of Frobenius, Journal of Number Theory 34 (1990), 198-209.

[ErGr72] P. Erdős and R. Graham, On a linear diophantine problem of Frobenius, Acta Arithmetica XXI (1972), 399-408. 
[Kh92] A. G. Khovanskii, Newton polyhedron, Hilbert polynomial, and sums of finite sets, Functional Analysis and Its Applications 26 (1992) 77-210.

[Nat72] Melvyn B. Nathanson, Sums of finite sets of integers, Amer. Math. Monthly 79 (1972) 10101012.

[Nat00] Melvyn B. Nathanson, Growth of sumsets in abelian semigroups. Semigroup Forum 61 (2000), $149-153$.

[NaRu02] Melvyn B. Nathanson and Imre Z. Ruzsa, Polynomial growth of sumsets in abelian semigroups Journal de théorie des nombres de Bordeaux, 14 (2002), 553-560.

[Ru09] Imre Ruzsa, Sumsets and structure, Combinatorial number theory and additive group theory. Birkhäuser Verlag, Basel, (eds. A. Geroldinger and Imre Ruzsa) (2009), 77-210.

[SaChe07] Svetoslav Savchev and Fang Chen, Long zero-free sequences in finite cyclic groups, Discrete Math. 307 (2007), 2671-2679.

[SaChe17] Svetoslav Savchev and Fang Chen, An inverse problem about minimal zero-sum sequences over finite cyclic groups, J. Number Theory 177 (2017), 381-427.

[SiTi03] R. Simpson and R. Tijdeman, Multi-dimensional versions of a Theorem of Fine and Wilf and a Formula of Sylvester, Proceedings of the American Mathematical Society, 131 (2003), 1661-1671.

[Sy1884] J. Sylvester, Mathematical questions, with their linear solutions. Educational times, 41 (1884), 21.

[WCC11] Jian-Dong Wu, Feng-Juan Chen and Yong-Gao Chen, On the structure of the sumsets, Discrete Math. 311 (2011), 408-412.

AG: Département de mathématiques et de statistique, Université de Montréal, CP 6128 succ. Centre-Ville, Montrál, QC H3C 3J7, Canada; and Department of Mathematics, University College London, Gower Street, London WC1E 6BT, England.

E-mail address: andrew@dms.umontreal.ca

GS: Mathematical Institute, University of Oxford, Andrew Wiles Building, RadCliffe Observatory Quarter, Woodstock Road, Oxford, OX2 6GG, UK.

E-mail address: george.shakan@gmail.com 